Original Research

\title{
A Point System Table to Estimate the Risk of Cardiovascular Events in Patients with Hypertension and Left Ventricular Hypertrophy: The LIFE Study
}

Darcy A Hille ${ }^{1}$, Sverre E Kjeldsen ${ }^{2,}{ }^{3}$, Stevo Julius ${ }^{3}$, Kristian Wachtell ${ }^{4}$, Peter M Okin ${ }^{4}$, Richard B Devereux ${ }^{4}{ }^{*}$

1. Merck \& Co., Inc., North Wales, PA, USA; E-Mail: darcy hille@merck.com

2. University of Oslo, Ullevaal Hospital, Departments of Cardiology, Oslo, Norway; E-Mail: s.e.kjeldsen@medisin.uio.no

3. University of Michigan Medical Center, Division of Cardiovascular Medicine, Ann Arbor, MI, USA; E-mail: sjulius@med.umich.edu

4. Weill Cornell Medicine, Greenberg Division of Cardiology, 525 East 68th Street, New York, NY, USA; E-Mails: kristian@wachtell.net; pokin@med.cornell.edu; rbdevere@med.cornell.edu

* Correspondence: Richard B. Devereux; E-Mail: rbdevere@med.cornell.edu

Academic Editor: Giuseppe Cocco

Special Issue: Geriatric Cardiac Diseases

OBM Geriatrics

2022, volume 6 , issue 1

doi:10.21926/obm.geriatr.2201189
Received: December 30, 2021

Accepted: January 24, 2022

Published: February 24, 2022

\begin{abstract}
We aimed to assess some readily available patient characteristics such as urinary albumin excretion as predictors of a primary composite event of myocardial infarction, cerebral stroke, and cardiovascular death among patients with hypertension and left ventricular hypertrophy, and develop risk algorithms for the outcomes. A "Cox proportional hazards" model identified the baseline variables that significantly affected the occurrence of the composite endpoint in 9193 elderly hypertensive patients with left ventricular hypertrophy in the Losartan Intervention For Endpoint reduction in hypertension (LIFE) study. We developed a risk point table, which assigned points to various risk factors, including the important age-gender interaction term, by adapting the point system algorithm developed from Framingham data.
\end{abstract}

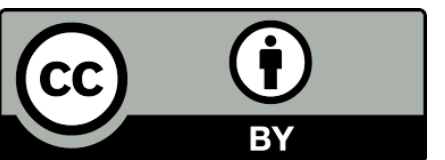

(C) 2022 by the author. This is an open access article distributed under the conditions of the Creative Commons by Attribution License, which permits unrestricted use, distribution, and reproduction in any medium or format, provided the original work is correctly cited. 
The Cox model identified 12 predictors of risk for the endpoint. Points for age were calculated separately for females and males due to the age-gender interaction. The risk estimates that were defined by total points were compared to those that were determined by the Cox model, and the weighted $\mathrm{k}$ value for the agreement was 0.89 . Thus, several patient characteristics predicted cardiovascular events in patients with hypertension and left ventricular hypertrophy, with age, gender, and urinary albumin excretion being the most important. The risk point table is an intuitive method to compare the relative contribution of the cardiovascular risk predictors and explain complicated statistical models such as the interaction term to patients and their physicians.

\section{Keywords}

Blood pressure; hypertension; left ventricular hypertrophy; prediction model; risk algorithms for outcomes; urinary albumin excretion

\section{Introduction}

The Losartan Intervention For Endpoint reduction in hypertension (LIFE) Study compared effects of losartan-based therapy with atenolol-based therapy on cardiovascular events in 9193 patients with hypertension and left ventricular hypertrophy. The design, baseline characteristics, and main results of the LIFE study have been previously described in other publications [1-3].

The primary endpoint was a composite of cardiovascular death, myocardial infarction, and stroke, with a mean follow-up time of 4.8 years. The statistical analysis of the primary endpoint was based on survival analysis models. The event rate was 508/4605 (11.0\%) in the losartan group and $588 / 4588(12.8 \%)$ in the atenolol group, with a hazard ratio of 0.87 (95\% Cl: 0.77 to 0.98$)$.

Investigators of the LIFE study [4] assessed patient characteristics, including urinary albumin excretion, which was not included in traditional cardiovascular risk scores, as predictors of cardiovascular events in patients with hypertension and left ventricular hypertrophy, and developed risk algorithms for the outcomes. The urine albumin/creatinine (UAC) ratio had a highly rightskewed distribution which precluded the use of t-tests or other parametric statistical tests such as univariate or multivariate linear regression to determine the significance of associations. This was corrected by the log transformation of the UAC ratio. Univariate and multivariate analyses identified baseline variables that significantly influenced the primary composite endpoint and its components. The multivariate analysis used a Cox regression model with a stepwise selection process. A risk score was developed based on the coefficients of risk factors from the multivariate analysis. This score was validated internally using naïve and jack-knife procedures, checked for discrimination and calibration, and compared with Framingham coronary heart disease and other risk scores. The risk score performed equally well or even better than the Framingham coronary heart disease risk score [5] in predicting the primary endpoint.

In the multivariate model described above, 12 variables significant affected the development of 1096 composite endpoints, including an age-sex interaction term. The risk score was described as follows: Risk score composite $=0.365 \times \log$ UAC ratio (per 10-fold) +0.476 (if current smoker) + 0.536 (if history of stroke or transient ischemic attack (TIA)) +0.474 (if history of diabetes) +0.646 
(if history of atrial fibrillation) +0.387 (if history of ischemic heart disease) $+0.115 \times$ total cholesterol (per mmol/l) - 0.258 (if exercise $>30$ min twice/week) $+0.116 \times$ LVH by Sokolow-Lyon voltage (SL) (per $10 \mathrm{~mm})+0.086 \times \mathrm{LVH}$ by Cornell product $(\mathrm{CP})($ per $1000 \mathrm{~mm} \cdot \mathrm{ms})$. Additional terms were added to the risk score for women $(0.786 \times$ [age-65]/10) and men $(0.434 \times$ [age-65]/10+0.703).

The approximate mean age ( 65 years) of patients in the study was subtracted from age to center the interaction term and facilitate interpretation of the parameter coefficients. Though this risk score performed well, it was rather complex. To use this score, the estimate of 5-year risks of the composite endpoint for a patient would be:

$$
\hat{p}=1-0.9044^{\exp (\text { Risk Score-7.07) }}
$$

Although the use of this risk score could be aided by an online calculator, it would be unlikely to be used in clinical practice. Besides, the score contained an interaction term (age $x$ gender). Despite the centering of the interaction, interpretation of the coefficients was difficult.

In this study, we aimed to assess the same easily available patient characteristics such as urinary albumin excretion as predictors of cardiovascular events in patients with hypertension and left ventricular hypertrophy and develop risk algorithms as a risk score point system table for the outcomes. A Cox proportional-hazards model identified baseline variables that could significantly impact the occurrence of the primary composite endpoint. A risk point table was developed, which assigned points to various levels of risk factors by adopting the point system algorithm developed by Sullivan et al. [6] based on Framingham data to include an interaction term.

\section{Materials and Methods}

The LIFE study assessed 9193 patients with hypertension and LVH on electrocardiograms based on either Cornell Product and/or Sokolow-Lyon criteria. Approximately 50\% of the patients had been previously treated for hypertension. Previous medication was discontinued, and all patients were given a single-blinded placebo run-in for 1-2 weeks. The criteria for qualification included resting hypertension of 160-200 $\mathrm{mmHg}$ systolic and/or 95-115 mmHg diastolic, with an average of $174 / 98 \mathrm{mmHg}$. Approximately 54\% of the patients were women; the mean body mass index was 28 $\mathrm{kg} / \mathrm{m}^{2}$, the mean resting heart rate was 74 beats/min, $16 \%$ were smokers, $14 \%$ had isolated systolic hypertension, and $13 \%$ had type-2 diabetes mellitus. Most of the patients (92\%) were of Caucasian ethnicity, as they were mainly from the Nordic Countries such as Denmark, Finland, Iceland, Norway, and Sweden, with 817 patients from the UK and 1707 patients from the USA. Framingham Risk Score averaged 0.224 at baseline, based on data from 945 participating study centers. Apart from hypertension and LVH, the patients were mostly healthy, although 25 reported some history of cardiovascular disease.

To facilitate the use of the risk score, a points table was developed, which assigned points to various levels of the risk factors, including interaction terms, by adopting the point system algorithm developed by Sullivan et al. [6]. The point system algorithm was applied according to the following procedure. 


\subsection{Estimate Parameters of the Multivariate Model}

A Cox regression model was applied to identify significant independent predictors of risk for the composite endpoint. To facilitate the interpretation of an age-sex interaction, age was recorded separately for males and females.

\subsection{Categories and Reference Values were Created for Each Factor}

Categories were created for each factor. For continuous factors, the categories were initially chosen based on the distribution of values and adjusted for clinical relevance and ease of interpretation.

\subsection{One Factor was Chosen as the Reference}

As age was chosen as the reference covariate, points for age were calculated first. Two years, or two regression units, represented one point.

\subsection{The Number of Points for Each Factor was Determined Based on the Factor's Scale and Size of Coefficient Relative to the Reference Coefficient}

We developed a table that presented the regression coefficients, chosen categories, reference values, base reference values, and the calculated points for each factor.

\subsection{Risk Associated with the Point Total was Determined}

The final step was to determine the risk associated with the total points. To check the agreement, the risk estimates from the points system and the Cox model were calculated for each patient in the LIFE study. The agreement by risk categories in $5 \%$ increments was presented with weighted $\mathrm{k}$ statistics and $95 \%$ confidence intervals.

\subsection{Ethics Statement}

The LIFE study was approved by ethical committees of all participating clinical centers. The participants signed written informed consent. The study was performed according to the Declaration of Helsinki. A written protocol was followed. The study was chaired by an academic steering committee and was overseen by an independent data and safety monitoring board. Data of the LIFE study are included in the Blood Pressure Lowering Treatment Trialists' Collaboration (BPLTTC), which is the world's largest database of data from individuals in hypertension RCTs. The data that supported the findings of the present study can be obtained from the corresponding author (RBD) upon dire need. The LIFE Study can be accessed from the URL https://www.clinicaltrials.gov with the unique identifier NCT00338260. 


\section{Results}

\subsection{Estimation of the Parameters of the Multivariable Model}

A Cox regression model (as described in the Methods section) identified age, sex, history of smoking, log UAC ratio, total cholesterol, history of stroke, diabetes, atrial fibrillation, ischemic heart disease, degree of left ventricular hypertrophy, exercise, and age-sex interaction as significant independent predictors of risk for the composite endpoint (Table 1). To facilitate interpretation of the interaction, age was converted into two terms: age of males and age of females. These were used instead of the age-sex interaction term.

Table 1 Primary Composite Endpoint ( $n=9193,1096$ cardiovascular events*).

\begin{tabular}{llll}
\hline Variable & $\begin{array}{l}\text { Regression } \\
\text { Coefficient }\end{array}$ & $\begin{array}{l}\text { Hazard Ratio } \\
(95 \% \mathrm{Cl})\end{array}$ & $\begin{array}{l}\text { Baseline Mean } \\
\text { or Proportion }\end{array}$ \\
\hline Age (males) & 0.0434 & $1.04(1.03-1.06)$ & 30.4007 \\
Age (females) & 0.0786 & $1.08(1.07-1.10)$ & 36.5410 \\
Male sex & 2.9886 & $19.86(5.32-74.19)$ & 0.4601 \\
Urinary albumin:creatinine ratio (per 10-fold) & 0.3654 & $1.44(1.32-1.58)$ & 0.2344 \\
Current smoker & 0.4763 & $1.61(1.39-1.86)$ & 0.1631 \\
Prior stroke/transient ischemic attack & 0.5356 & $1.71(1.45-2.01)$ & 0.0792 \\
Diabetes & 0.4740 & $1.61(1.38-1.86)$ & 0.1300 \\
Atrial fibrillation & 0.6458 & $1.91(1.55-2.35)$ & 0.0352 \\
Prior ischemic heart disease & 0.3870 & $1.47(1.28-1.69)$ & 0.1598 \\
Total cholesterol (per mmol/L) & 0.00298 & $1.003(1.002-1.004)$ & 233.5114 \\
Exercise >30 min twice per week & -0.2578 & $0.77(0.68-0.87)$ & 0.5176 \\
LVH by Sokolow-Lyon (per 10 units) & 0.1157 & $1.12(1.06-1.19)$ & 29.9956 \\
LVH by Cornell product (per 1000 units) & 0.0862 & $1.09(1.04-1.15)$ & 28.2347 \\
\hline
\end{tabular}

$\mathrm{LVH}=$ left ventricular hypertrophy

*Composite of myocardial infarction, stroke, and cardiovascular death

The risk was estimated by the following equation:

$$
\text { Risk estimate } \hat{p}=1-S_{0}(\mathrm{t})^{\exp \left(\sum \beta X-\sum \beta \bar{X}\right)}
$$

where $\mathrm{SO}(\mathrm{t})$ was the average survival at time $t, \beta$ represents the Cox regression coefficients, $X$ represents the individual's values of risk factors and $\bar{X}$ represents the means (or proportions) of the risk factors. Here, S0 (5 years) $=0.9044$, and the value of the model at the means (or proportions) $\sum \beta \bar{X}=7.07$.

Thus,

$$
\hat{p}=1-0.9044^{\exp (\Sigma \beta X-7.07)}
$$

where $\sum \beta X$ represents the risk score as mentioned before. The point score method produced an estimate of the risk score but was easier to calculate. 


\subsection{Creation of Categories and Reference Values for Each Factor}

Categories need to be created for each factor. For continuous variables, the categories were initially chosen based on the distribution of the values and adjusted for clinical relevance and ease of interpretation. Each category had a reference value, Wij, which was generally the midpoint of the category. The base or reference value WiREF was usually chosen from the category that reflected the lowest risk, as the base category was assigned 0 points in the scoring system. In the case of yes/no variables, "no" was chosen as the base value.

\subsection{Choosing One Factor as the Reference}

Since age was chosen as the reference covariate, the points for age were calculated first. Two years, or two regression units, represented one point. If $B$ represents one point in regression units and $\beta_{\text {age }}$ of female $=0.07856$, then $B=0.158$. After the establishment of the $B$ value, points for each category were calculated simply using the regression coefficient, the width of the category, and $B$, using the formula: Points $=(\beta \mathrm{i} *(\mathrm{Wij}-\mathrm{WiREF})) / \mathrm{B}$.

\subsection{Determination of the Number of Points of Each Factor Based on the Factor's Scale and Size of Coefficient Relative to the Reference Coefficient}

Table 2 presents the regression coefficients, chosen categories, reference values, the base reference values, and the calculated points for each factor. The simplified table is presented in Table 3.

Table 2 Factor Categories, Reference Values, and Points.

\begin{tabular}{llllll}
\hline \multirow{2}{*}{ Risk Factor } & Categories & $\begin{array}{l}\text { Reference } \\
\text { value (Wij) }\end{array}$ & $\begin{array}{l}\text { Regression } \\
\text { Coefficient } \\
\beta \mathrm{i}\end{array}$ & $\begin{array}{l}\beta \mathrm{i}(\text { Wij- } \\
\text { WiREF })\end{array}$ & $\begin{array}{l}\text { Pointsij }= \\
\beta \mathrm{i}(\text { Wij- } \\
\text { WiREF)/B }\end{array}$ \\
\hline Age (women) & & & 0.0786 & & \\
& $<57$ & 56 & & -0.31424 & -2 \\
& 57 to 59 & 58 & -0.15712 & -1 \\
& 59 to 61 & $60=$ W1REF & 0 & 0 \\
61 to 63 & 62 & 0.15712 & 1 \\
63 to 65 & 64 & 0.31424 & 2 \\
65 to 67 & 66 & 0.47136 & 3 \\
67 to 69 & 68 & 0.62848 & 4 \\
69 to 71 & 70 & 0.7856 & 5 \\
71 to 73 & 72 & 0.94272 & 6 \\
73 to 75 & 74 & 1.09984 & 7 \\
75 to 77 & 76 & 1.25696 & 8 \\
$\geq 77$ & 78.5 & 1.45336 & 9
\end{tabular}

Age (men)

0.0434

$\begin{array}{ll}<57 & 56 \\ 57 \text { to } 59 & 58\end{array}$

$\begin{array}{ll}-0.1736 & -1 \\ -0.0868 & -1\end{array}$


OBM Geriatrics 2022; 6(1), doi:10.21926/obm.geriatr.2201189

$\begin{array}{llll}59 \text { to } 61 & 60=\text { W2REF } & 0 & 0 \\ 61 \text { to } 63 & 62 & 0.0868 & 1 \\ 63 \text { to } 65 & 64 & 0.1736 & 1 \\ 65 \text { to } 67 & 66 & 0.2604 & 2 \\ 67 \text { to } 69 & 68 & 0.3472 & 2 \\ 69 \text { to } 71 & 70 & 0.434 & 3 \\ 71 \text { to } 73 & 72 & 0.5208 & 3 \\ 73 \text { to } 75 & 74 & 0.6076 & 4 \\ 75 \text { to } 77 & 76 & 0.6944 & 4 \\ \geq 77 & 78 & 0.8029 & 5\end{array}$

Gender

$\begin{array}{llll}\text { Female } & 0=\text { W3REF } & 0 & 0 \\ \text { Male } & 1 & 2.98857 & 19\end{array}$

Urinary

0.36539

albumin:

creatinine

ratio (per 10-

fold)

$\begin{array}{ll}<-0.5 & -0.65 \\ -0.5 \text { to }-0.3 & -0.4 \\ -0.3 \text { to } 0.3 & 0=\text { W4REF } \\ 0.3 \text { to } 0.7 & 0.5 \\ 0.7 \text { to } 1.1 & 0.9 \\ 1.1 \text { to } 1.3 & 1.2 \\ \geq 1.3 & 1.69\end{array}$

$-0.2375 \quad-2$

$-0.14616 \quad-1$

$0 \quad 0$

$0.182695 \quad 1$

$0.328851 \quad 2$

$0.438468 \quad 3$

$0.617509 \quad 4$

Diabetes

$\begin{array}{ll}\text { No } & 0=\text { W5REF } \\ \text { Yes } & 1\end{array}$

0.47402

$\begin{array}{ll}0 & 0 \\ 0.47402 & 3\end{array}$

History of No $0=$ W6REF

atrial

fibrillation

Yes 1

0.64582

$\begin{array}{ll}0 & 0 \\ 0.64582 & 4\end{array}$

Ischemic heart disease

$\begin{array}{ll}\text { No } & 0=\text { W7REF } \\ \text { Yes } & 1\end{array}$

0.38703

$\begin{array}{ll}0 & 0 \\ 0.38703 & 2\end{array}$


OBM Geriatrics 2022; 6(1), doi:10.21926/obm.geriatr.2201189

$\begin{array}{llll}\text { No } & 0=\text { W8REF } & 0 & 0 \\ \text { Yes } & 1 & 0.53563 & 3\end{array}$

Smoker

0.4763

$\begin{array}{llll}\text { No } & 0=\text { W9REF } & 0.0000 & 0 \\ \text { Yes } & 1 & 0.4763 & 3\end{array}$

Total

Cholesterol

0.00298

$\begin{array}{ll}<160 & 151.5 \\ 160 \text { to } 200 & 180=\text { W } 10 \text { REF } \\ 200 \text { to } 240 & 220 \\ 240 \text { to } 280 & 260 \\ >=280 & 312\end{array}$

$-0.25784$

$\begin{array}{llll}\text { No } & 0=\text { W11REF } & 0 & 0 \\ \text { Yes } & 1 & -0.25784 & -2\end{array}$

$\begin{array}{llllll}\text { Sokolow-Lyon } & <20 & 15=\text { W12REF } & 0.01157 & & \\ & 20-35 & 27.5 & 0 & 0 \\ & \geq 35 & 46.25 & 0.144638 & 1 \\ & & & 0.361594 & 2\end{array}$

Cornell

Product

0.00862

$\begin{array}{llll}<20 & 16.665= & & \\ & \text { W12REF } & 0 & 0 \\ 20-35 & 30 & 0.114948 & 1 \\ \geq 35 & 50.75 & 0.293813 & 2\end{array}$

TIA = transient ischemic attack

Table 3 Categories of Risk Factors and Points

Risk Factor

Gender

Age

\section{Categories Points}

$\begin{array}{ll}\text { female } & 0 \\ \text { male } & 19\end{array}$

\begin{tabular}{|c|c|c|c|c|}
\hline & & & (female) & (male) \\
\hline 55 & $<$ & 57 & -2 & -1 \\
\hline 57 & & 59 & -1 & -1 \\
\hline 59 & & 61 & 0 & 0 \\
\hline
\end{tabular}




\begin{tabular}{lllllll}
\hline 61 & to & 63 & 1 & 1 \\
63 & to & 65 & 2 & 1 \\
65 & to & 67 & 3 & 2 \\
67 & to & 69 & 4 & 2 \\
69 & to & 71 & 5 & 3 \\
71 & to & 73 & 6 & 3 \\
73 & to & 75 & 7 & 4 \\
75 & to & 77 & 8 & 4 \\
80 & $>=$ & 77 & 9 & 5
\end{tabular}

log urine:creatinine ratio

$\begin{array}{llll}-0.8 & < & -0.5 & -2 \\ -0.5 & \text { to } & -0.3 & -1 \\ -0.3 & \text { to } & 0.3 & 0 \\ 0.3 & \text { to } & 0.7 & 1 \\ 0.7 & \text { to } & 1.1 & 2 \\ 1.1 & \text { to } & 1.3 & 3 \\ 2.08 & >= & 1.3 & 4\end{array}$

Diabetes

$\begin{array}{ll}\text { No } & 0 \\ \text { Yes } & 3\end{array}$

Atrial fibrillation

No $\quad 0$

Yes 4

Ischemic heart disease

$\begin{array}{ll}\text { No } & 0 \\ \text { Yes } & 2\end{array}$

Prior stroke/transient ischemic attack

$\begin{array}{ll}\text { No } & 0 \\ \text { Yes } & 3\end{array}$

Smoker

$\begin{array}{ll}\text { No } & 0 \\ \text { Yes } & 3\end{array}$

Total Cholesterol

$\begin{array}{llll}143 & < & 160 & -1 \\ 160 & \text { to } & 200 & 0 \\ 200 & \text { to } & 240 & 1 \\ 240 & \text { to } & 280 & 2 \\ 344 & >= & 280 & 3\end{array}$

Exercise $>30$ min $2 x$ week

$\begin{array}{ll}\text { No } & 0 \\ \text { Yes } & -2\end{array}$

Sokolow-Lyon

$10<20 \quad 0$ 


\begin{tabular}{lllll}
\hline & 20 & to & 35 & 1 \\
& 57.5 & $>=$ & 35 & 2 \\
Cornell Product & & & & \\
& 8.33 & $<$ & 25 & 0 \\
& 25 & to & 35 & 1 \\
& 66.5 & $>=$ & 35 & 2 \\
\hline
\end{tabular}

\subsection{Determination of Risk Associated with the Point Total}

The final step was to determine the risk associated with the point totals. The risk estimate was calculated as $\hat{p}=1-S_{0}(\mathrm{t})^{\exp \left(\sum \beta X-\sum \beta \bar{X}\right)}$, where so (5 years) $=0.9044$ and $\sum \beta \bar{X}=7.07$. To calculate the risk estimate, we need the value of $\sum \beta X$. The value of $\mathrm{B}^{*}$ (point total) is equivalent to that of $\sum \beta X$, with the addition of non-zero reference values of continuous variables, such as age, cholesterol content, Sokolow-Lyon value, and Cornell product. Due to the interaction between age and gender, this was calculated separately according to the gender.

For females: $\sum \beta X \approx 0.0434(60)+0.00298(180)+0.011571(15)+0.00862(14.165)+$ $B$ (point total)

For males: $\sum \beta X \approx 5.0 .0786(60)+0.00298(180)+0.011571(15)+0.00862(14.165)+$ $B$ (point total)

This can be simplified as follows:

$$
\begin{aligned}
& \text { For females: } \sum \beta X \approx 5.546+(\text { point total }) \\
& \text { For males: } \sum \beta X \approx 3.437+(\text { point total })
\end{aligned}
$$

And finally,

$$
\begin{gathered}
\hat{p}_{\text {females }}=1-0.9044^{\exp (5.546+B(\text { point total })-7.07))} \\
\hat{p}_{\text {males }}=1-0.9044^{\exp (3.437+B(\text { point total })-7.07))}
\end{gathered}
$$

For females, the possible point totals ranged from -7 to 33 , while for males, they ranged from 13 to 48 . However, there would be few individuals with extremely high point totals, so we capped the point totals at 16 and 30 when the 5 -year estimate of the risk of the primary composite endpoint was about $25 \%$. Due to the low sample size of high-risk individuals for nearly every factor, the higher estimates of risk may not be reliable. Table 4 summarizes these estimates.

Table 4 Points and Calculated 5-year Risk for the Primary Composite Endpoint.

\begin{tabular}{llll}
\hline Females & & Males & \\
Point Total & Estimate of Risk & Point Total & Estimate of Risk \\
\hline-7 & 0.0073 & 13 & 0.0203 \\
-6 & 0.0085 & 14 & 0.0237 \\
-5 & 0.0099 & 15 & 0.0277 \\
-4 & 0.0116 & 16 & 0.0323 \\
\hline
\end{tabular}




\begin{tabular}{llll}
\hline-3 & 0.0136 & 17 & 0.0377 \\
-2 & 0.0159 & 18 & 0.0439 \\
-1 & 0.0185 & 19 & 0.0512 \\
0 & 0.0217 & 20 & 0.0597 \\
1 & 0.0253 & 21 & 0.0694 \\
2 & 0.0295 & 22 & 0.0808 \\
3 & 0.0345 & 23 & 0.0938 \\
4 & 0.0402 & 24 & 0.1089 \\
5 & 0.0469 & 25 & 0.1262 \\
6 & 0.0547 & 26 & 0.1461 \\
7 & 0.0637 & 27 & 0.1687 \\
8 & 0.0741 & 28 & 0.1944 \\
9 & 0.0861 & 29 & 0.2235 \\
10 & 0.1000 & 30 & $>0.25$ \\
11 & 0.1160 & & \\
12 & 0.1344 & & \\
13 & 0.1554 & & \\
14 & 0.1793 & & \\
15 & 0.2064 & & \\
16 or more & $>0.24$ & & \\
\hline
\end{tabular}

The following clinical examples illustrate the relationship between the estimated risk from the point system and that from the Cox regression model.

\subsubsection{Clinical Example 1}

A 72-year-old female with total cholesterol of $234 \mathrm{mg} / \mathrm{dL}$, non-diabetic, non-smoker, SokolowLyon value of $16.5 \mathrm{~mm}$, Cornell Product of $27.5 \mathrm{~mm} \cdot \mathrm{ms}$, not an exerciser, no history of ischemic heart disease, atrial fibrillation, or cerebrovascular accident (stroke)/transient ischemic attack. Her point total and estimate of risk would be:

\begin{tabular}{lll}
\hline Risk factor & Value & Points \\
\hline Age & 72 & 6 \\
Log urine albumin/creatinine ratio & 0.52395 & 1 \\
Total Cholesterol & 234 & 1 \\
Sokolow-Lyon & 16.5 & 0 \\
Cornell Product & 27.5 & 1 \\
& & \\
& Point Total & 9 \\
& Estimate of Risk & 0.086 \\
\hline
\end{tabular}

The risk estimate based on the Cox model would be computed as follows: 


$$
\begin{aligned}
& \sum_{i=1}^{p} \beta_{i} X_{i}=0.0786(72)+02.9886(0)+0.365(0.52359)+0.476(0)+0.536(0)+0.474(0) \\
&+ 0.646(0)+0.387(0)+0.00298(234)-.258(0)+0.0116(16.5) \\
&+ 0.0086(27.5)=6.97 \\
& \hat{p}=1-S_{0}(t)^{\exp \left(\sum \beta X-\sum \beta \tilde{X}\right)}=1-0.9044^{\exp (6.97-7.07)}=.087
\end{aligned}
$$

The points system gives a 5 -year risk of the composite endpoint of $8.6 \%$, whereas the Cox model estimates the risk at $8.7 \%$.

\subsubsection{Clinical Example 2}

A 59-year-old male smoker with total cholesterol of $232 \mathrm{mg} / \mathrm{dL}$, non-diabetic, Sokolov-Lyon value of $38.5 \mathrm{~mm}$, Cornell Product of $31.8 \mathrm{~mm} \cdot \mathrm{ms}$, not an exerciser, history of ischemic heart disease but no history of atrial fibrillation or cerebrovascular accident (stroke)/transient ischemic attack. His point total and estimate of risk would be:

\begin{tabular}{lll}
\hline Risk factor & Value & Points \\
\hline Gender & Male & 19 \\
Age & 59 & 0 \\
Log urine albumin/creatinine ratio & 0.1505 & 0 \\
Smoker & Yes & 3 \\
Ischemic Heart Disease & Yes & 2 \\
Total Cholesterol & 232 & 1 \\
Sokolow-Lyon & 28.5 & 1 \\
Cornell Product & 31.8 & 1 \\
& & \\
& Point Total & 27 \\
& Estimate of Risk & 0.169 \\
\hline
\end{tabular}

The risk estimate based on the Cox model would be computed as follows:

$$
\begin{aligned}
\sum_{i=1}^{p} \beta_{i} X_{i}= & 0.0434(59)+2.9886(1)+0.365(0.1505)+0.476(1)+0.536(0)+0.474(0) \\
& +0.646(0)+0.387(1)+0.00298(232)-.258(0)+0.0116(28.5) \\
& +0.0086(31.8)=7.76 \\
& \hat{p}=1-S_{0}(t)^{\exp \left(\sum \beta X-\sum \beta \tilde{X}\right)}=1-0.9044^{\exp (7.76-7.07)}=0.182
\end{aligned}
$$

Thus, the points system gives a 5 -year risk of the composite endpoint of $16.9 \%$, whereas the Cox model estimates the risk at $18.2 \%$.

To check the agreement between the two models, the risk estimates from both were calculated for each patient in the LIFE study. The agreement by categories of risk in $5 \%$ increments is presented in Table 5. The weighted $\mathrm{k}$ statistic was 0.89 , with a $95 \%$ confidence interval of 0.88 and 0.90 . 
Table 5 Agreement Between the Risk Estimates based on the Points System and the Cox Model Was Calculated for Each Patient in the LIFE study.

\begin{tabular}{lllll}
\hline \multicolumn{5}{l}{ The 5-yr risk from the points table } \\
\hline The 5-yr risk from the Cox model & $<0.05$ & $0.05-0.10$ & $0.10-0.15$ & $\geq 0.15$ \\
$<0.05$ & 1599 & 175 & 0 & 0 \\
$0.05-0.10$ & 89 & 2791 & 180 & 1 \\
$0.10-0.15$ & 0 & 284 & 1457 & 218 \\
$\geq 0.15$ & 0 & 2 & 233 & 2164 \\
\hline
\end{tabular}

\section{Discussion}

The main finding of our study was that several demographic characteristics of our study population of $>9000$ participants with hypertension and left ventricular hypertrophy predicted the composite study endpoint of myocardial infarction, cerebral stroke, and cardiovascular death. The most important predictors in our study were age and gender with an important interaction, as well as urinary albumin excretion, which was assessed as log UAC ratio. We developed a point score system that represented the relative contributions of the investigated patients' characteristics. Thus, we could easily present an advanced statistical model for interested readers, including patients with hypertension or left ventricular hypertrophy and their physicians.

When we conducted the LIFE Study, we used common ECG criteria to diagnose patients with left ventricular hypertrophy among hypertensive patients in the age range of 55-80 years. The ECG criteria were described in the 2018 European Society of Cardiology and European Society of Hypertension guidelines, which have been recommended as the first-line screening tool for LVH and other cardiac diseases among people with hypertension. The patients were first given a placebo run-in to qualify for further participation based on elevated blood pressures within certain ranges. They were then randomized to compare the angiotensin receptor-blocker losartan with the $\beta-1$ selective blocker atenolol in a further double-blinded design, while still achieving the same level of blood pressure control during follow-up. The study was endpoint-driven, meaning that 1040 primary composite endpoints had to occur to achieve statistical power with the characteristics of the population, including a 5-year Framingham risk score of 0.221 [2, 5]. This led to an average follow-up period of 4.8 years. To achieve a high enough risk of events, an elderly population with an age range of $55-80$ years and a mean of 67 years was selected. The population had a body mass index (average of $28 \mathrm{~kg} / \mathrm{m}^{2}$ ) that was typical for hypertensive people in this age range from NorthEurope and the USA, while certain fractions of included patients with isolated systolic hypertension [7] and type-2 diabetes mellitus [8] contributed to the risk.

Other traditional risk factors, such as smoking habits, chronic and acute atrial fibrillation, blood lipid levels, physical exercise, left ventricular hypertrophy by ECG, and history of cardiovascular disease, also contributed to the risk in the LIFE population. However, the influence of these factors was somewhat less than those discussed above. We have previously [4] discussed why blood pressure levels, heart rate, and body mass index were not significant predictors of the primary composite endpoint in the LIFE Study.

Urinary albumin excretion in younger and elderly patients with hypertension and LVH has never been compared. The existing data apply to a largely elderly population in the age range of 55-80 
years, with a mean of 67 years at baseline. The rate of the primary endpoint in the LIFE Study was 26 events/1000 patient-years, which was not as high as that observed in the Swedish Trial on Old Patients with Hypertension [9]. Although the primary endpoint was the same, the participants in the Swedish trial were on average 9 years older and had higher blood pressures at the time of qualification and inclusion into the study. However, when we compared the data of our LIFE Study with the findings of Gueyffier et al. [10] in a summary study of nearly 25,000 patients of a similar profile, several statistically significant risk factors were observed to be similar.

The risk scores developed by us using the readily available patients' data in the LIFE Study precisely identified the cardiovascular outcomes with even better efficiency than Framingham score for coronary heart disease [5] and cerebral stroke [11] or Pocock score for cardiovascular death [12]. However, a key difference compared to other risk scores $[5,11,13-19]$ was that we included UAC score in the LIFE scores, as recommended in another study [20]. Urinary albumin excretion appeared to be a strong predictor of cardiovascular outcomes [21]. Also, the reduction in urinary albumin excretion during treatment with losartan could explain approximately $20 \%$ of the statistical difference in the study outcome between losartan and atenolol [22]. In this regard, the data of our LIFE study agreed with the findings of a study on Native Americans [23] and thus support more general use of urinary albumin excretion in generating risk models in cardiovascular research. Such a risk score may be important in the future design of intervention trials. However, the LIFE risk score may also apply to direct clinical assessment of the several millions of European and American patients with hypertension and left ventricular hypertrophy by ECG [24]

Certain limitations may apply when we compare the risk assessments of the LIFE Study with those deduced from the populations included in the Framingham and Pocock studies [5, 10, 12]. Particularly the populations included in those studies did not include patients with primarily left ventricular hypertrophy as diagnosed using ECG, or those in the elderly age range, compared to our study.

Our study also has certain advantages. All investigator-reported endpoints were adjudicated by an expert committee. Besides, the average follow-up was as long as 4.8 years. Also, to include enough patients from the USA, we had a study population of about 1000 more patients than initially planned. Moreover, we measured urinary albumin excretion both at baseline and at scheduled visits during follow-up. Furthermore, none of the patients were lost to follow-up in Scandinavia, while only 12 patients were lost to follow-up in the USA. Finally, approximately $75 \%$ of the randomized patients stayed on blinded medication throughout the entire duration of the study.

Initially, we developed a rather complex LIFE Study risk score and thought that it could be useful with the help of a web-based computer program [4]. We validated the initial LIFE score internally and compared it with the other scores as discussed above $[5,10,12]$. We found a high accuracy and precision. We did not validate the LIFE score in other samples of patients, as another large group of hypertensive elderly patients with LVH did not exist, which made it impossible to verify our model in a similar population.

In summary, we developed a risk point table that assigned points to various levels of risk factors, including interaction terms, by adopting the point system algorithm developed by Sullivan et al. [6]. The Cox model identified age, sex, smoking, log UAC ratio, total cholesterol, history of stroke, diabetes, atrial fibrillation, ischemic heart disease, degree of left ventricular hypertrophy, exercise, and age-sex interaction as significant predictors of risk for the composite endpoint. Points for age were calculated separately for females and males due to interaction. Points for age (55 to 80 years) 
ranged from -2 to 10 for females and from 5 to 11 for males, compared to just 4 points for elevated urinary albumin excretion. Risk estimates defined by point totals were compared to those determined by the Cox model, and the weighted $\mathrm{k}$ value for the agreement was 0.89 .

We established the protocol in 1993-94 and included systematic urinalysis for albumin excretion during all study visits. Thus, to the best of our knowledge, our study is the only one to present such data of patients with hypertension and LVH. Therefore, our risk model is also the only one to include the effect of urinary albumin excretion, which is powerful in this group of elderly patients. We measured the UAC ratios in spot urine samples of all patients during all study visits, when patients met in the morning hours, throughout the study ( $24 \mathrm{~h}$ after the latest intake of study medication). Thus, the measurements were highly standardized, as explained in our previous publication [21].

The angiotensin receptor-blocker losartan reduced urinary albumin excretion, as previously discussed (25). However, our prediction model aimed to be independent of specific drugs. Thus, as explained in the Materials and Methods, our prediction model was adjusted for study treatment in the multivariate analysis.

Left ventricular hypertrophy has for many years been intriguing clinicians [25, 26]. The use of the point table developed herein from LIFE data facilitates the assessment of left ventricular hypertrophy more widely when assessing risk and taking decisions related to aggressiveness of treatment of patients with hypertension.

\section{Conclusion}

In the LIFE study, the baseline characteristics of several participants predicted the composite event of myocardial infarction, cerebral stroke, and cardiovascular mortality in patients with hypertension and left ventricular hypertrophy. Age, gender, and urinary albumin excretion were the most important predictors. The point score table provides a genuine and simple method to compare the relative contributions of various risk predictors and provide information about a complicated statistical model, including interaction, to patients and their physicians.

\section{Author Contributions}

Professor Sverre E. Kjeldsen, MD, and Professor Stevo Julius, MD, were coordinators of the LIFE study and responsible for patient inclusions and follow-up in the Nordic Countries and in USA, respectively. Darcy $\mathrm{H}$. Hille, MS, has performed the statistical analyses and presented an abstract at the American College of Cardiology annual scientific meeting [27]. Kristian Wachtell, MD, was secretary of the echocardiographic sub-study. Professor Peter Okin, MD, was responsible for the EKG protocol. Professor Richard B. Devereux, MD, was responsible for the echocardiographic protocol and the echocardiographic reading center. All authors were responsible for reading and approving the final manuscript.

\section{Funding}

The Life Study was originally supported by Merck Sharp \& Dohme Corp., a subsidiary of Merck \& Co., Inc., Kenilworth, NJ, USA.. 


\section{Competing Interest}

Professor Sverre E. Kjeldsen, MD, has received lecture honoraria within the past 3 years from Getz Pharma, Merck Healthcare KGaA, Sanofi-Aventis and Vector-Intas. The other authors have declared that no competing interests exist.

\section{References}

1. Dahlöf B, Devereux R, de Faire U, Fyhrquist F, Hedner T, Ibsen H, et al. The Losartan Intervention For Endpoint reduction (LIFE) in Hypertension study: Rationale, design, and methods. Am J Hypertens. 1997; 10: 705-713.

2. Dahlöf B, Devereux RB, Julius S, Kjeldsen SE, Beevers G, de Faire U, et al. Characteristics of 9194 patients with left ventricular hypertrophy: The LIFE study. Hypertension. 1998; 32: 989-997.

3. Dahlöf B, Devereux RB, Kjeldsen SE, Julius S, Beevers G, de Faire U, et al. Cardiovascular morbidity and mortality in the Losartan Intervention For Endpoint reduction in hypertension study (LIFE): A randomised trial against atenolol. Lancet. 2002; 359: 995-1003.

4. Kjeldsen SE, Deverux RB, Hille D, Lyle PA, Dahlöf B, Julius S, et al. Predictors of cardiovascular events in patients with hypertensive and left ventricular hypertrophy: The LIFE Study. Blood Press. 2009; 18: 348-361.

5. Anderson KM, Wilson PW, Odell PM, Kannel WB. An updated coronary risk profile. A statement for health professionals. Circulation. 1991; 83: 356-362.

6. Sullivan LM, Massaro JM, d'Agostino Sr RB. Presentation of multivariate data for clinical use: The Framingham study risk score functions. Stat Med. 2004; 23: 1631-1660.

7. Dahlöf B, Lindholm LH, Hansson L, Scherstén B, Ekbom T, Wester PO. Morbidity and mortality in the Swedish Trial in Old Patients with Hypertension (STOP-Hypertension). Lancet. 1991; 338: 1281-1285.

8. Kjeldsen SE, Dahlöf B, Devereux RB, Julius S, Aurup P, Edelman J, et al. Effect of losartan on cardiovascular morbidity and mortality in patients with isolated systolic hypertension and left ventricular hypertrophy. A Losartan Intervention For Endpoint Reduction (LIFE) substudy. JAMA. 2002; 288: 1491-1498.

9. Lindholm LH, Ibsen H, Dahlöf B, Devereux RB, Beevers G, de Faire $U$, et al. Cardiovascular morbidity and mortality in patients with diabetes in the Losartan Intervention For Endpoint reduction in hypertension study (LIFE): A randomized trial against atenolol. Lancet. 2002; 359: 1004-1010.

10. Gueyffier F, Boissel JP, Pocock S, Boutitie F, Coope J, Cutler J, et al. Identification of risk factors in hypertensive patients: Contribution of randomized controlled trials through an individual patient database. Circulation. 1999; 100: e88-e94.

11. Wolf PA, D'Agostino RB, Belanger AJ, Kannel W. Probability of stroke: A risk profile from the Framingham Study. Stroke. 1991; 22: 312-318.

12. Pocock SJ, McCormack V, Gueyffier F, Boutitie F, Fagard RH, Boissel JP. A score for predicting risk of death from cardiovascular disease in adults with raised blood pressure, based on individual patient data from randomised controlled trials. BMJ. 2001; 323: 75-81.

13. Levy D, Garrison RJ, Savage DD, Kannel WB, Castelli WP. Prognostic implications of echocardiographically determined left ventricular mass in the Framingham Heart Study. N Engl J Med. 1990; 322: 1561-1566. 
14. D'Agostino Sr RB, Vasan RS, Pencina MJ, Wolf PA, Cobain M, Massaro JM, et al. General cardiovascular risk profile for use in primary care: The Framingham Heart Study. Circulation. 2008; 117; 743-753.

15. Liao Y, Cooper RS, McGee DL, Mensah GA, Ghali JK. The relative effects of left ventricular hypertrophy, coronary artery disease, and ventricular dysfunction on survival among black adults. JAMA. 1995; 273: 1592-1597.

16. Conroy RM, Pyorala K, Fitzgerald AP, Sans S, Menotti A, De Backer G, et al. Estimation of tenyear risk of fatal cardiovascular disease in Europe: The SCORE project. Eur Heart J. 2003; 24: 987-1003.

17. Hippisley-Cox J, Coupland C, Vinogradova Y, Robson J, May M, Brindle P. Derivation and validation of QRISK, a new cardiovascular disease risk score for the United Kingdom: Prospective open cohort study. BMJ. 2007; 335: 136.

18. Ridker PM, Buring JE, Rifai N, Cook NR. Development and validation of improved algorithms for the assessment of global cardiovascular risk in women: The Reynolds risk score. JAMA. 2007; 297: 611-619.

19. Woodward $M$, Brindle $P$, Tunstall-Pedoe $H$. Adding social deprivation and family history to cardiovascular risk assessment: The ASSIGN score from the Scottish Heart Health Extended Cohort (SHHEC). Heart. 2007; 93: 172-176.

20. Weir MR. Microalbuminuria and cardiovascular disease. Clin J Am Soc Nephrol. 2007; 2: 581590

21. Wachtell K, Ibsen H, Olsen MH, Borch-Johnsen K, Lindholm LH, Mogensen CE, et al. Albuminuria and cardiovascular risk in hypertensive patients with left ventricular hypertrophy: The LIFE study. Ann Intern Med. 2003; 139: 901-906.

22. Ibsen $H$, Wachtell K, Olsen MH, Borch-Johnsen K, Lindholm LH, Mogensen CE, et al. Does albuminuria predict cardiovascular outcome on treatment with losartan versus atenolol in patients with hypertension and left ventricular hypertrophy? A LIFE substudy. J Hypertens. 2004; 22: 1805-1811.

23. Lee ET, Howard BV, Wang W, Welty TK, Galloway JM, Best LG, et al. Prediction of coronary heart disease in a population with high prevalences of diabetes and albuminuria: The Strong Heart Study. Circulation. 2006; 113: 2897-2905.

24. Dahlöf B, Burke TA, Krobot K, Carides GW, Edelman JM, Devereux RB, et al. Population impact of losartan use on stroke in the European Union (EU): Projections from the Losartan Intervention For Endpoint reduction in hypertension (LIFE) study. J Hum Hypertens. 2004; 18 : 367-373.

25. Koren MJ, Devereux RB, Casale PN, Savage DD, Laragh JH. Relation of left ventricular mass and geometry to morbidity and mortality in uncomplicated essential hypertension. Ann Intern Med. 1991; 114: 345-352.

26. Otterstad JE, Smiseth O, Kjeldsen SE. Hypertensive left ventricular hypertrophy: Pathophysiology, assessment and treatment. Blood Press. 1996; 5: 5-15.

27. Hille DA, Kjeldsen SE, Dahlöf B, Edelman JM, Devereux RB. A point system table to estimate the risk of cardiovascular events in patients with hypertension and left ventricular hypertrophy. The Losartan Intervention For Endpoint Reduction in Hypertension (LIFE) Study. JACC. 2009; 53: A214. 


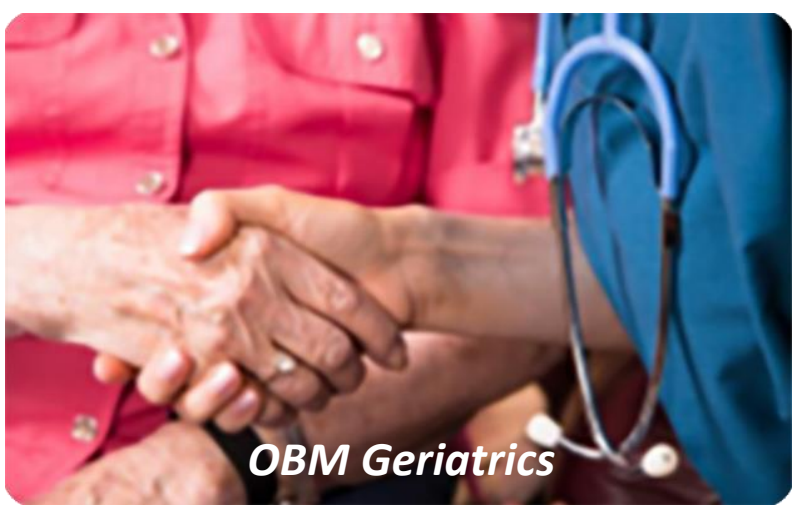

Enjoy OBM Geriatrics by:

1. Submitting a manuscript

2. Joining in volunteer reviewer bank

3. Joining Editorial Board

4. Guest editing a special issue

For more details, please visit:

http://www.lidsen.com/journals/geriatrics 